\title{
A Memetic Approach for Improving Minimum Cost of Economic Load Dispatch Problems
}

\author{
Jinho Kim, Chang Seob Kim, and Zong Woo Geem \\ Department of Energy IT, Gachon University, Seongnam 461-701, Republic of Korea \\ Correspondence should be addressed to Zong Woo Geem; geem@gachon.ac.kr
}

Received 21 October 2013; Revised 16 December 2013; Accepted 7 January 2014; Published 23 February 2014

Academic Editor: Swagatam Das

Copyright (C) 2014 Jinho Kim et al. This is an open access article distributed under the Creative Commons Attribution License, which permits unrestricted use, distribution, and reproduction in any medium, provided the original work is properly cited.

\begin{abstract}
Economic load dispatch problem is a popular optimization problem in electrical power system field, which has been so far tackled by various mathematical and metaheuristic approaches including Lagrangian relaxation, branch and bound method, genetic algorithm, tabu search, particle swarm optimization, harmony search, and Taguchi method. On top of these techniques, this study proposes a novel memetic algorithm scheme combining metaheuristic algorithm and gradient-based technique to find better solutions for an economic load dispatch problem with valve-point loading. Because metaheuristic algorithms have the strength in global search and gradient-based techniques have the strength in local search, the combination approach obtains better results than those of any single approach. A bench-mark example of 40 generating-unit economic load dispatch problem demonstrates that the memetic approach can further improve the existing best solutions from the literature.
\end{abstract}

\section{Introduction}

Economic load dispatch (ELD) problem is a classical form of optimization problems and has been one of the most important decision-making processes in the operation of electrical power systems. The total system-wide generation cost is generally defined as the objective function of ELD problem. The equality and inequality power system constraints are embedded in ELD formulation, such as power balance and generation limits of each generating unit's output capacity. ELD problem has been thought of as a mathematically complex and highly nonlinear optimization problem, especially in larger systems. For many decades, many algorithms have been presented to solve the optimization problem of ELD. First, conventional deterministic approaches which resort to mathematical gradient information have been developed to obtain the minimum cost of ELD problem. To overcome the limitations of those deterministic algorithms in realsystem applications which are basically associated with the simplification of mathematical formulation, a variety of evolutionary frameworks that employ metaheuristic computational intelligence have explored their capabilities to search optimal solution of ELD problem with little abbreviation of original formulation.

Many gradient-based and enumeration-based deterministic approaches such as Lagrangian relaxation and linear, nonlinear, and dynamic programming techniques have been applied to find the optimal solution of ELD problem [1-7]. They are suitable, however, only when the problem satisfies certain conditions (complex calculus-based gradient should be derived or formulation can be approximated in terms of linearity and/or convexity), and therefore this mathematical requirement has limited the extensive applications of these deterministic algorithms in real ELD problem. In addition, extensive search algorithms which explore all the solution space such as dynamic programming $[2,3]$, therefore, have been employed in ELD problem to tackle the issue of the simplification in the mathematical formulation of ELD. Due to the so-called "curse of dimensionality" and local optimality, however, these approaches hardly give solutions with higher level of satisfaction in the real-world applications.

For recent decades, therefore, a variety of artificial intelligence approaches which are dependent upon heuristic and 
stochastic search scheme have been intensively adopted in ELD problem. Many studies have aimed to overcome the shortcomings of the conventional deterministic algorithms and to investigate the efficiency and applicability of the algorithms in ELD problem. For example, genetic-based different types of algorithms (GA) [8-15], a variety of evolutionary programming-based algorithms (EP) [16-19], particle swarm optimization (PSO) with its variations [20-28], tabu search (TS) [29], Taguchi method [30], bioinspired optimization algorithms [31-34], harmony search (HS) [35, 36], and hybrid methods combining two or more metaheuristic algorithms [37-42] have been applied to explore the capability of stochastic artificial intelligence algorithms to solve ELD problem so far.

Up until now, we can see that several well-developed metaheuristic algorithms have shown a higher level of applicability in ELD problem with fine performance [43-53]. In spite of the acceptable level of performance in some algorithms of ELD problem, we have found that a memetic approach for ELD problem to combine the well-developed evolutionary frameworks with gradient-based local search algorithm can provide an opportunity for better solutions. Through this memetic combination, it can be seen that the minimum cost of ELD problem can be further improved because metaheuristic algorithm's weak local search performance is reinforced by calculus-based method and calculusbased method's weak global search performance is reinforced by metaheuristic algorithm.

This paper is organized as follows. Mathematical formulation of ELD problems and recent metaheuristic algorithms to solve ELD problem are described in Section 2. In Section 3, memetic implementations for those algorithms are explored to integrate gradient-based local search into the evolutionary frameworks for the solution of ELD. Simulation results and discussions on the algorithm proposed are provided in Section 4, followed by conclusion and future investigation in Section 5 .

\section{Problem Description: Economic Load Dispatch with Valve-Point Loading}

The ELD problem can be stated as to determine the optimal set of individual generating units' generation outputs minimizing the objective function(s) as well as satisfying both the equality and inequality constrains. The ELD can, therefore, be mathematically formulated as a continuous variable optimization problem. The objective function to be minimized can be defined as the system-wide generation cost across all the generators. Equality constraint of ELD is a power balancing equation where total power supply of all the generators is equal to the total system demand plus system loss. In addition, individual generators' generation output should be in-between its minimum and maximum generation capacity, and this condition is imposed as the inequality constraint for each generator's output in ELD problem. The mathematical formulation of the generic ELD problem can, therefore, be described as follows:

$$
\begin{aligned}
\text { Min } C= & \sum_{i \in I} F_{i}\left(P_{i}\right)=\sum_{i \in I}\left\lfloor a_{i}+b_{i} P_{i}+c_{i} P_{i}^{2}\right\rfloor \\
\text { subject to } & \sum_{i \in I} P_{i}=D+L \\
& \underline{P_{i}} \leq P_{i} \leq \bar{P}_{i},
\end{aligned}
$$

where $C$ is system-wide total generating units' cost function; $F_{i}$ is generation cost function of generating unit-i; $P_{i}$ is generation output of generating unit- $i ; a_{i}, b_{i}$, and $c_{i}$ are cost coefficients of generating unit $i$; $I$ is index set of generating units in the system; $D$ is total system demand; $L$ is total system loss; $\underline{P_{i}}, \overline{P_{i}}$ are minimum and maximum generation output of generating unit- $i$.

The system loss in the transmission lines, $L$, can be presented as a quadratic form of generating units' output generations as follows:

$$
L=\sum_{i \in I} \sum_{j \in I} P_{i} B_{i j} P_{j}+\sum_{i \in I} P_{i} B_{0 i}+B_{00}
$$

where $B_{i j}$ is the $i j$ th element of loss coefficient matrix, $B_{0 i}$ is $i$ th element of loss coefficient vector, and $B_{00}$ is $i$ th element of loss coefficient constant.

Moreover, more practical consideration of ELD problem requires an inclusion of valve-point loading effects in ELD problem, and the mathematical formulation of ELD with valve-point loading can be rephrased as follows:

$$
\begin{aligned}
\operatorname{Min} C & =\sum_{i \in I} F_{i}\left(P_{i}\right) \\
& =\sum_{i \in I}\left\lfloor a_{i}+b_{i} P_{i}+c_{i} P_{i}^{2}+\left|e_{i} \cdot \sin \left(f_{i} \cdot\left(\underline{P_{i}}-P_{i}\right)\right)\right|\right\rfloor,
\end{aligned}
$$

where $e_{i}$ and $f_{i}$ are nonsmooth fuel cost coefficients of generating unit- $i$.

Many gradient-based and enumeration-based deterministic approaches have been applied to find the optimal solution of ELD problem, and they are suitable only when the problem satisfies certain conditions. Extensive search algorithms have been employed in ELD problem to tackle the issue of the simplification in the mathematical formulation of ELD. These approaches hardly give solutions with higher level of satisfaction in the real-world applications due to the curse of dimensionality and local optimality. For recent decades, many artificial intelligence approaches have been intensively adopted in ELD problem to investigate the efficiency and applicability of the algorithms in ELD problem. In ELD problem, although several algorithms for solving economic dispatch are well developed up until now, we have found that memetic crossover between gradient-based algorithm and metaheuristic methodology can provide opportunity of better solutions for ELD. The memetic algorithm is based on the characteristic of the meme in human culture, which is the basic unit of knowledge that can be modified, combined with other ones, and generating new ones to propagate in 
the communities [63]. Under the optimization problems like ELD, the memetic algorithm considers complex structures of conventional metaheuristic algorithms and simple gradientbased optimization operators, where the interactions of evolutionary and local search algorithm lead to an efficient solution method that is able to solve ELD problem more effectively. In this study, therefore, a memetic approach to combine stochastic metaheuristic algorithms with gradientbased local search is presented to explore the opportunity of better solution for ELD problem.

Many metaheuristic approaches using population-based evolutionary frameworks have been applied in ELD problem in order to find the optimal solution. The populationbased metaheuristic algorithms for ELD primarily resort to iterated procedures for initializing, competing, and updating of population in reaching optimal solution(s), and, therefore, the general solution steps of the metaheuristic algorithms for ELD can be described as shown in Algorithm 1.

Depending upon the specific individual evolutionary framework in a metaheuristic algorithm, the initialization, competition, and update of population process for ELD in the algorithm can vary. Some algorithms employ a random initialization for the parent population, while the others attempt to use a better quality one as a starting population by using a refined heuristic. In the iterated competing and updating processes for ELD, the respective metaheuristic algorithms adopt their own computational intelligence operators based upon their functional advantages over other ones. For example, genetic and evolutionary upgrade operators are used in genetic and evolutionary algorithms. Social patterns in the behaviors of animals are modeled in particle swarm optimization, honey bee mating, and firefly algorithm. Also, physical phenomenon-based population improvement is explored in harmony search, tabu search, and simulated annealing. As for the termination, the iterated processes in the algorithms stop based upon their own termination criteria, which are typically defined as the maximum number of iterations and/or minimum extent of the improvement of the fitness value(s) for ELD.

In recent ELD studies using metaheuristic algorithms, several biogeography-based and musical improvisation methodologies are presented to find the optimal solution of ELD problem [33, 34, 36, 64]. In [33, 34], a firefly algorithm (FA) and a honey bee mating optimization (HBMO) algorithm have been developed to solve nonconvex ELD problem with valve loading effect, respectively, and they have shown a higher level of performance than conventional metaheuristic algorithms. In addition, a variation of pattern search (PS) methods and a population-variance harmony search (HS) algorithm have also been presented to solve the constrained ELD problem, in [36, 64], respectively. Two algorithms have also demonstrated their better capabilities in ELD applications than existing relevant approaches.

\section{Memetic Implementations for ELD Problem}

Memetic computing is a branch in computer science which regards complicate structures as the combination of heterogeneous operators, named memes, whose evolutionary interactions contribute to intelligent structures for problem solving $[65,66]$.

For recent decades, memetic algorithms have been widely applied in the large-scale complex optimization problems. The problem of balance between global and local search, that is, balance between computational intelligence and gradientbased search algorithm, has been explored under a multiobjective optimization setting in $[67,68]$. In [69], a memetic algorithm is presented to analyze the evolutionary artificial neural network for training of a medical application. For a large-scale combinatorial optimization problem, a parallel memetic algorithm with selective local search is proposed in [70]. In [71-73], memetic algorithms are applied to solve scheduling and planning problems such as vehicle routing and path planning. A fast adaptive memetic algorithm for a design of controller of engineering drives is presented in [74], and a compact memetic differential algorithm has been developed for a robot control in [75]. In [76], a differential evolution-based hybrid algorithm is provided to solve the optimization problem in aerodynamic design.

In order to explore the opportunity of better solution in ELD problem using the memetic combination proposed in this study, we have basically considered three metaheuristic algorithms: harmony search (HS), firefly algorithm (FA), and honey bee mating optimization (HBMO), which show the highest level of performances among all the existing relevant population-based metaheuristic approaches in the most recent ELD problem literatures [33, 34, 36]. HS algorithm was originally inspired by the improvisation process of Jazz musicians. The algorithm uses a novel stochastic derivative which utilizes the experiences of musicians in Jazz improvisation and can be applicable to optimization problem. Instead of the gradient-based inclination information of an objective function, the stochastic derivative of the algorithm gives a probability to be selected for each value of a decision variable. In [36], the evolution of the population-variance over successive generations in HS was analyzed and the algorithm showed that it can easily take care of solving nonconvex ELD problems along with different constraints. The FA was based on the idealized behavior of the flashing characteristics of fireflies such that the brightness or light intensity of a firefly is affected or determined by the landscape of the objective function to be optimized. In [33], a FA for solving nonsmooth economic dispatch with various constraints was presented and the results are compared with recent ELD solution methods. The HBMO algorithm is based upon the social characteristics of the honey bee in working together in a highly structured social order. In [34], a method to improve the mating process of $\mathrm{HBMO}$ combining the $\mathrm{HBMO}$ with a chaotic local search was proposed to overcome the disadvantage of the conventional algorithm that may miss the optimum and provide a near optimum solution in a limited runtime period. Table 1 shows the minimum cost of ELD problem using the most recent three metaheuristic algorithms in $[33,34,36]$ (i.e., HS, FA, and HBMO), with the same test system of 40 generating units.

Based upon the result in Table 1, therefore, we have proposed a memetic combination of the most recent HS, 
Step 1. Initialization of the population for the parent generation

Step 2. Iterations

Step 2.1. Creation of the population for the new generation

Step 2.2. Competition among populations

Step 2.3. Population update

Step 3. Termination

Algorithm 1: The general solution steps of the metaheuristic algorithms for ELD.

TABLE 1: Minimum costs of ELD problem from three different algorithms.

\begin{tabular}{|c|c|c|c|c|c|c|}
\hline \multirow{2}{*}{ Number } & \multicolumn{3}{|c|}{ Generation (MW) } & \multicolumn{3}{|c|}{ Cost $(\$)$} \\
\hline & HS & FA & HMBO & HS & FA & HMBO \\
\hline 1 & 110.8312 & 110.8099 & 110.8018 & 925.6191 & 925.2642 & 925.1296 \\
\hline 2 & 110.8234 & 110.8059 & 110.8000 & 925.4891 & 925.1976 & 925.0998 \\
\hline 3 & 97.3999 & 97.4023 & 97.3999 & 1190.5485 & 1190.5949 & 1190.5485 \\
\hline 4 & 179.7331 & 179.7332 & 179.7331 & 2143.5503 & 2143.5524 & 2143.5503 \\
\hline 5 & 88.9932 & 92.7070 & 87.7998 & 726.2998 & 787.1211 & 706.5003 \\
\hline 6 & 140.0000 & 140.0000 & 140.0000 & 1596.4643 & 1596.4643 & 1596.4643 \\
\hline 7 & 259.6006 & 259.6004 & 259.5997 & 2612.9019 & 2612.8983 & 2612.8846 \\
\hline 8 & 284.6143 & 284.6004 & 284.5997 & 2780.1031 & 2779.8502 & 2779.8368 \\
\hline 9 & 284.5997 & 284.6004 & 284.5997 & 2798.2312 & 2798.2440 & 2798.2303 \\
\hline 10 & 130.0000 & 130.0028 & 130.0000 & 2502.0650 & 2502.1290 & 2502.0650 \\
\hline 11 & 168.7998 & 168.8008 & 94.0000 & 2959.4585 & 2959.4811 & 1893.3054 \\
\hline 12 & 168.7998 & 168.8008 & 94.0000 & 2977.4549 & 2977.4776 & 1908.1668 \\
\hline 13 & 214.7598 & 214.7606 & 214.7598 & 3792.0703 & 3792.0901 & 3792.0700 \\
\hline 14 & 394.2794 & 304.5204 & 394.2794 & 6414.8612 & 5149.7187 & 6414.8604 \\
\hline 15 & 304.5191 & 394.2801 & 394.2794 & 5171.1965 & 6436.6047 & 6436.5863 \\
\hline 16 & 394.2794 & 394.2801 & 394.2794 & 6436.5870 & 6436.6047 & 6436.5863 \\
\hline 17 & 489.2794 & 489.2801 & 489.2794 & 5296.7114 & 5296.7265 & 5296.7107 \\
\hline 18 & 489.2794 & 489.2801 & 489.2794 & 5288.7658 & 5288.7809 & 5288.7652 \\
\hline 19 & 511.2796 & 511.2817 & 511.2794 & 5540.9342 & 5540.9797 & 5540.9292 \\
\hline 20 & 511.2794 & 511.2817 & 511.2794 & 5540.9099 & 5540.9597 & 5540.9092 \\
\hline 21 & 523.2867 & 523.2793 & 523.2794 & 5071.4381 & 5071.2898 & 5071.2897 \\
\hline 22 & 523.2836 & 523.2793 & 523.2807 & 5071.3753 & 5071.2898 & 5071.3156 \\
\hline 23 & 523.2794 & 523.2832 & 523.2794 & 5057.2237 & 5057.3002 & 5057.2231 \\
\hline 24 & 523.2794 & 523.2832 & 523.2794 & 5057.2237 & 5057.3002 & 5057.2231 \\
\hline 25 & 523.2794 & 523.2793 & 523.2794 & 5275.0891 & 5275.0886 & 5275.0885 \\
\hline 26 & 523.2794 & 523.2793 & 523.2794 & 5275.0891 & 5275.0886 & 5275.0885 \\
\hline 27 & 10.0000 & 10.0000 & 10.0000 & 1140.5240 & 1140.5240 & 1140.5240 \\
\hline 28 & 10.0000 & 10.0000 & 10.0000 & 1140.5240 & 1140.5240 & 1140.5240 \\
\hline 29 & 10.0000 & 10.0000 & 10.0000 & 1140.5240 & 1140.5240 & 1140.5240 \\
\hline 30 & 91.2184 & 87.8008 & 87.7999 & 762.9889 & 706.5149 & 706.5001 \\
\hline 31 & 190.0000 & 189.9989 & 190.0000 & 1643.9913 & 1643.9869 & 1643.9913 \\
\hline 32 & 190.0000 & 189.9989 & 190.0000 & 1643.9913 & 1643.9869 & 1643.9913 \\
\hline 33 & 190.0000 & 189.9989 & 190.0000 & 1643.9913 & 1643.9869 & 1643.9913 \\
\hline 34 & 164.9179 & 164.8036 & 164.8015 & 1587.5968 & 1585.6100 & 1585.5733 \\
\hline 35 & 164.8672 & 164.8036 & 194.3928 & 1541.0193 & 1539.9348 & 1985.3690 \\
\hline 36 & 164.8786 & 164.8036 & 200.0000 & 1541.2137 & 1539.9348 & 2043.7270 \\
\hline 37 & 110.0000 & 110.0000 & 110.0000 & 1220.1661 & 1220.1661 & 1220.1661 \\
\hline 38 & 110.0000 & 110.0000 & 110.0000 & 1220.1661 & 1220.1661 & 1220.1661 \\
\hline 39 & 110.0000 & 110.0000 & 110.0000 & 1220.1661 & 1220.1661 & 1220.1661 \\
\hline 40 & 511.2795 & 511.2794 & 511.2794 & 5540.9320 & 5540.9299 & 5540.9292 \\
\hline Sum & 10500.0000 & 10500.0000 & 10500.0000 & 121415.4560 & 121415.0522 & 121412.5704 \\
\hline
\end{tabular}


TABLE 2: Generating units data for test system.

\begin{tabular}{|c|c|c|c|c|c|c|c|}
\hline Number & $\underline{P}$ & $\bar{P}$ & $a$ & $b$ & $c$ & $e$ & $f$ \\
\hline 1 & 36 & 114 & 0.0069 & 6.73 & 94.705 & 100 & 0.084 \\
\hline 2 & 36 & 114 & 0.0069 & 6.73 & 94.705 & 100 & 0.084 \\
\hline 3 & 60 & 120 & 0.02028 & 7.07 & 309.54 & 100 & 0.084 \\
\hline 4 & 80 & 190 & 0.00942 & 8.18 & 369.03 & 150 & 0.063 \\
\hline 5 & 47 & 97 & 0.0114 & 5.35 & 148.89 & 120 & 0.077 \\
\hline 6 & 68 & 140 & 0.01142 & 8.05 & 222.33 & 100 & 0.084 \\
\hline 7 & 110 & 300 & 0.00357 & 8.03 & 287.71 & 200 & 0.042 \\
\hline 8 & 135 & 300 & 0.00492 & 6.99 & 391.98 & 200 & 0.042 \\
\hline 9 & 135 & 300 & 0.00573 & 6.6 & 455.76 & 200 & 0.042 \\
\hline 10 & 130 & 300 & 0.00605 & 12.9 & 722.82 & 200 & 0.042 \\
\hline 11 & 94 & 375 & 0.00515 & 12.9 & 635.2 & 200 & 0.042 \\
\hline 12 & 94 & 375 & 0.00569 & 12.8 & 654.69 & 200 & 0.042 \\
\hline 13 & 125 & 500 & 0.00421 & 12.5 & 913.4 & 300 & 0.035 \\
\hline 14 & 125 & 500 & 0.00752 & 8.84 & 1760.4 & 300 & 0.035 \\
\hline 15 & 125 & 500 & 0.00708 & 9.15 & 1728.3 & 300 & 0.035 \\
\hline 16 & 125 & 500 & 0.00708 & 9.15 & 1728.3 & 300 & 0.035 \\
\hline 17 & 220 & 500 & 0.00313 & 7.97 & 647.85 & 300 & 0.035 \\
\hline 18 & 220 & 500 & 0.00313 & 7.95 & 649.69 & 300 & 0.035 \\
\hline 19 & 242 & 550 & 0.00313 & 7.97 & 647.83 & 300 & 0.035 \\
\hline 20 & 242 & 550 & 0.00313 & 7.97 & & 300 & 0.035 \\
\hline 21 & 254 & 550 & 0.00298 & 6.63 & 785.96 & 300 & 0.035 \\
\hline 22 & 254 & 550 & 0.00298 & 6.63 & 785.96 & 300 & 0.035 \\
\hline 23 & 254 & 550 & 0.00284 & 6.66 & 794.53 & 300 & 0.035 \\
\hline 24 & 254 & 550 & 0.00284 & 6.66 & 794.53 & 300 & 0.035 \\
\hline 25 & 254 & 550 & 0.00277 & 7.1 & 801.32 & 300 & 0.035 \\
\hline 26 & 254 & 550 & 0.00277 & 7.1 & 801.32 & 300 & 0.035 \\
\hline 27 & 10 & 150 & 0.52124 & 3.33 & & 120 & 0.077 \\
\hline 28 & 10 & 150 & 0.52124 & 3.33 & 1055.1 & 120 & 0.077 \\
\hline 29 & 10 & 150 & 0.52124 & 3.33 & 1055.1 & 120 & 0.077 \\
\hline 30 & 47 & 97 & 0.0114 & 5.35 & 148.89 & 120 & 0.077 \\
\hline 31 & 60 & 190 & 0.0016 & 6.43 & 222.92 & 150 & 0.063 \\
\hline 32 & 60 & 190 & 0.0016 & 6.43 & 222.92 & 150 & 0.063 \\
\hline 33 & 60 & 190 & 0.0016 & 6.43 & 222.92 & 150 & 0.063 \\
\hline 34 & 90 & 200 & 0.0001 & 8.95 & 107.87 & 200 & 0.042 \\
\hline 35 & 90 & 200 & 0.0001 & 8.62 & 116.58 & 200 & 0.042 \\
\hline 36 & 90 & 200 & 0.0001 & 8.62 & 116.58 & 200 & 0.042 \\
\hline 37 & 25 & 110 & 0.0161 & 5.88 & 307.45 & 80 & 0.098 \\
\hline 38 & 25 & 110 & 0.0161 & 5.88 & 307.45 & 80 & 0.098 \\
\hline 39 & 25 & 110 & 0.0161 & 5.88 & 307.45 & 80 & 0.098 \\
\hline 40 & 242 & 550 & 0.00313 & 7.97 & 647.83 & 300 & 0.035 \\
\hline
\end{tabular}

FA, and HBMO algorithms with gradient-based local search operator to improve the minimum cost of the ELD problem. The memetic approach to metaheuristic algorithms for ELD problem combines a local search operator with a metaheuristic algorithm, and it is a basically population-based metaheuristic which enables an evolutionary framework to search local solutions in a gradient manner within a certain generation cycle. As the memetic approach blends together a population-based computational intelligence algorithm and local search method, the memetic algorithm for ELD problem
TABLE 3: Results for Test Case 1.

\begin{tabular}{|c|c|c|c|c|}
\hline \multirow{2}{*}{ Number } & \multicolumn{2}{|c|}{ Generation (MW) } & \multicolumn{2}{|c|}{ Cost $(\$)$} \\
\hline & HS [36] & M_HS & HS [36] & M_HS \\
\hline 1 & 110.8312 & 110.8322 & 925.6191 & 925.6363 \\
\hline 2 & 110.8234 & 110.8234 & 925.4891 & 925.4891 \\
\hline 3 & 97.3999 & 97.3999 & 1190.5485 & 1190.5484 \\
\hline 4 & 179.7331 & 179.7331 & 2143.5503 & 2143.5503 \\
\hline 5 & 88.9932 & 88.9932 & 726.2998 & 726.2998 \\
\hline 6 & 140.0000 & 140.0000 & 1596.4643 & 1596.4643 \\
\hline 7 & 259.6006 & 259.6005 & 2612.9019 & 2612.9009 \\
\hline 8 & 284.6143 & 284.6142 & 2780.1031 & 2780.1021 \\
\hline 9 & 284.5997 & 284.5996 & 2798.2312 & 2798.2303 \\
\hline 10 & 130.0000 & 130.0000 & 2502.0650 & 2502.0650 \\
\hline 11 & 168.7998 & 168.7998 & 2959.4585 & 2959.4585 \\
\hline 12 & 168.7998 & 168.7998 & 2977.4549 & 2977.4549 \\
\hline 13 & 214.7598 & 214.7598 & 3792.0703 & 3792.0699 \\
\hline 14 & 394.2794 & 394.2793 & 6414.8612 & 6414.8603 \\
\hline 15 & 304.5191 & 304.5195 & 5171.1965 & 5171.1976 \\
\hline 16 & 394.2794 & 394.2793 & 6436.5870 & 6436.5862 \\
\hline 17 & 489.2794 & 489.2794 & 5296.7114 & 5296.7108 \\
\hline 18 & 489.2794 & 489.2794 & 5288.7658 & 5288.7652 \\
\hline 19 & 511.2796 & 511.2794 & 5540.9342 & 5540.9303 \\
\hline 20 & 511.2794 & 511.2794 & 5540.9099 & 5540.9092 \\
\hline 21 & 523.2867 & 523.2866 & 5071.4381 & 5071.4355 \\
\hline 22 & 523.2836 & 523.2835 & 5071.3753 & 5071.3728 \\
\hline 23 & 523.2794 & 523.2793 & 5057.2237 & 5057.2231 \\
\hline 24 & 523.2794 & 523.2793 & 5057.2237 & 5057.2231 \\
\hline 25 & 523.2794 & 523.2793 & 5275.0891 & 5275.0885 \\
\hline 26 & 523.2794 & 523.2793 & 5275.0891 & 5275.0885 \\
\hline 27 & 10.0000 & 10.0000 & 1140.5240 & 1140.5240 \\
\hline 28 & 10.0000 & 10.0000 & 1140.5240 & 1140.5240 \\
\hline 29 & 10.0000 & 10.0000 & 1140.5240 & 1140.5240 \\
\hline 30 & 91.2184 & 91.2184 & 762.9889 & 762.9891 \\
\hline 31 & 190.0000 & 190.0000 & 1643.9913 & 1643.9913 \\
\hline 32 & 190.0000 & 190.0000 & 1643.9913 & 1643.9913 \\
\hline 33 & 190.0000 & 190.0000 & 1643.9913 & 1643.9913 \\
\hline 34 & 164.9179 & 164.9179 & 1587.5968 & 1587.5964 \\
\hline 35 & 164.8672 & 164.8672 & 1541.0193 & 1541.0191 \\
\hline 36 & 164.8786 & 164.8786 & 1541.2137 & 1541.2135 \\
\hline 37 & 110.0000 & 110.0000 & 1220.1661 & 1220.1661 \\
\hline 38 & 110.0000 & 110.0000 & 1220.1661 & 1220.1661 \\
\hline 39 & 110.0000 & 110.0000 & 1220.1661 & 1220.1661 \\
\hline 40 & 511.2795 & 511.2793 & 5540.9320 & 5540.9292 \\
\hline Sum & 10500.0000 & 10500.0000 & 121415.4560 & 121415.4525 \\
\hline
\end{tabular}

can provide a better opportunity for the optimal solution by combining two individual robust operators, which have higher capability of global search and local search, in the solution space, respectively. Therefore, the general structure of the memetic algorithm for ELD can be rephrased as shown in Algorithm 2 to mimetically associate gradientbased search algorithm with conventional population-based metaheuristic. 
Step 1. Initialization of the population for the parent generation

Step 2. Iterations

Step 2.1. Creation of the population for the new generation

Step 2.2. Competition among population

Step 2.3. Population update

Step 3. Gradient-based local search

Step 4. Termination

Algorithm 2: The general structure of the memetic approach to metaheuristic for ELD.

\section{Gradient-Based Local Search Algorithm}

For the gradient-based local search algorithm, this study adopted BFGS (Broyden-Fletcher-Goldfarb-Shanno) method [77], which can solve nonlinear optimization problems by eliminating technical constraints. The solution steps of the BFGS method are as follows.

Step 1. Identify an initial feasible solution, $\mathbf{x}^{k=0}=$ $\left(x_{1}^{0}, x_{2}^{0}, \ldots, x_{n}^{0}\right)$.

Step 2. Calculate the searching direction, $\mathbf{d}^{k}$.

Step 3. Calculate a new solution, $\mathbf{x}^{k+1}=\mathbf{x}^{k}+\beta^{k} \mathbf{d}^{k}$, where $\beta^{k}$ is the step size which minimizes $f\left(\mathbf{x}^{k}+\beta^{k} \mathbf{d}^{k}\right)$.

Step 4. If convergence criterion is not satisfied, set $k=k+1$ and go to Step 2.

The searching direction in Step 2 can be obtained by various methods such as steepest descent, conjugate gradient, quasi-Newton, and Newton methods. Out of various approaches, this study chose the quasi-Newton method, named BFGS, because it is one of the most powerful techniques. The BFGS method emulates the inverse Hessian matrix instead of directly calculating it as follows:

$$
\begin{gathered}
\mathbf{d}^{k+1}=\mathbf{G}^{k+1} \nabla f\left(\mathbf{x}^{k+1}\right), \\
\nabla f(\mathbf{x})=\left[\frac{\partial f}{\partial \mathbf{x}}\right]=\left(\frac{\partial f}{\partial x_{1}}, \frac{\partial f}{\partial x_{2}}, \ldots, \frac{\partial f}{\partial x_{n}}\right)^{T}, \\
\mathbf{G}^{k+1}=\mathbf{G}^{k}+\left(\frac{1+\left(\mathbf{Y}^{k}\right)^{T} \mathbf{G}^{k} \mathbf{Y}^{k}}{\left(\mathbf{Y}^{k}\right)^{T} \mathbf{S}^{k}}\right) \frac{\mathbf{S}^{k}\left(\mathbf{S}^{k}\right)^{T}}{\left(\mathbf{S}^{k}\right)^{T} \mathbf{Y}^{k}} \\
-\frac{\mathbf{Y}^{k}\left(\mathbf{S}^{k}\right)^{T} \mathbf{G}^{k}+\mathbf{G}^{k} \mathbf{S}^{k}\left(\mathbf{Y}^{k}\right)^{T}}{\left(\mathbf{Y}^{k}\right)^{T} \mathbf{S}^{k}} \\
\mathbf{Y}^{k+1}=\nabla f\left(\mathbf{x}^{k+1}\right)-\nabla f\left(\mathbf{x}^{k}\right), \\
\mathbf{S}^{k+1}=\mathbf{x}^{k+1}-\mathbf{x}^{k} .
\end{gathered}
$$

The convergence criterion in this study is the relative error as follows:

$$
\left|\frac{f\left(\mathbf{x}^{k}\right)-f\left(\mathbf{x}^{k+1}\right)}{f\left(\mathbf{x}^{k}\right)}\right|<\varepsilon .
$$

The BFGS method, which is a calculus-based technique, has the advantages over metaheuristic algorithms in terms of (1) fast convergence (it takes less than one second in this study), (2) good local search performance, and (3) having no algorithm parameter requirement (e.g., crossover rate in GA and harmony memory considering rate in HS). However, it also has the disadvantages over metaheuristic algorithms in terms of (1) less global search performance and even (2) divergence [77-79].

\section{Numerical Experiments and Discussions}

5.1. Test System. The numerical test system used to explore the applicability of memetic algorithms in ELD problem in this study consists of forty generating units with valve-point loading effects, and the total system demand is $10,500 \mathrm{MW}$ [18]. Table 2 shows the data of the forty generating units with valve-point for the test system. In order to compare the performance proposed in this paper to those of existing metaheuristic only algorithms, we have adopted the same test system that is used in previous studies [18].

5.2. Test Case 1: Memetic Implementation with Harmony Search. First, we have implemented a memetic approach using HS algorithm. The best minimum generation cost of ELD for the given test system using HS reported until now is $\$ 121,415.4560$ [36], and each generator's output generation at the optimal point is presented in Table 3. The better minimum cost of ELD using a memetic approach to HS (M_HS) proposed in this study, however, can be obtained as $\$ 121,415.4525$, and the results are also provided in Table 3.

5.3. Test Case 2: Memetic Implementation with Firefly Algorithm. As a second experiment, we have carried out a memetic implementation using FA. In [33], the best minimum generation cost of ELD for the same test system using FA has been reported as $\$ 121,415.0522$, and each generator's output generation at this point is given in Table 4. However, the better minimum cost of ELD can be obtained using a memetic approach to FA (M_FA) presented in this paper as $\$ 121,414.9137$, and the results are also provided in Table 4. 
TABle 4: Results for Test Case 2.

\begin{tabular}{|c|c|c|c|c|}
\hline \multirow{2}{*}{ Number } & \multicolumn{2}{|c|}{ Generation (MW) } & \multicolumn{2}{|c|}{ Cost $(\$)$} \\
\hline & FA [33] & M_FA & FA [33] & M_FA \\
\hline 1 & 110.8099 & 110.8341 & 925.2642 & 925.6675 \\
\hline 2 & 110.8059 & 110.8059 & 925.1976 & 925.1976 \\
\hline 3 & 97.4023 & 97.3999 & 1190.5949 & 1190.5484 \\
\hline 4 & 179.7332 & 179.7330 & 2143.5524 & 2143.5501 \\
\hline 5 & 92.7070 & 92.7077 & 787.1211 & 787.1319 \\
\hline 6 & 140.0000 & 140.0000 & 1596.4643 & 1596.4643 \\
\hline 7 & 259.6004 & 259.5996 & 2612.8983 & 2612.8845 \\
\hline 8 & 284.6004 & 284.5997 & 2779.8502 & 2779.8377 \\
\hline 9 & 284.6004 & 284.5996 & 2798.2440 & 2798.2303 \\
\hline 10 & 130.0028 & 130.0000 & 2502.1290 & 2502.0650 \\
\hline 11 & 168.8008 & 168.7998 & 2959.4811 & 2959.4583 \\
\hline 12 & 168.8008 & 168.7999 & 2977.4776 & 2977.4566 \\
\hline 13 & 214.7606 & 214.7598 & 3792.0901 & 3792.0700 \\
\hline 14 & 304.5204 & 304.5195 & 5149.7187 & 5149.6989 \\
\hline 15 & 394.2801 & 394.2792 & 6436.6047 & 6436.5857 \\
\hline 16 & 394.2801 & 394.2792 & 6436.6047 & 6436.5857 \\
\hline 17 & 489.2801 & 489.2799 & 5296.7265 & 5296.7230 \\
\hline 18 & 489.2801 & 489.2799 & 5288.7809 & 5288.7772 \\
\hline 19 & 511.2817 & 511.2793 & 5540.9797 & 5540.9291 \\
\hline 20 & 511.2817 & 511.2793 & 5540.9597 & 5540.9091 \\
\hline 21 & 523.2793 & 523.2793 & 5071.2898 & 5071.2898 \\
\hline 22 & 523.2793 & 523.2793 & 5071.2898 & 5071.2898 \\
\hline 23 & 523.2832 & 523.2794 & 5057.3002 & 5057.2231 \\
\hline 24 & 523.2832 & 523.2794 & 5057.3002 & 5057.2231 \\
\hline 25 & 523.2793 & 523.2793 & 5275.0886 & 5275.0886 \\
\hline 26 & 523.2793 & 523.2793 & 5275.0886 & 5275.0886 \\
\hline 27 & 10.0000 & 10.0000 & 1140.5240 & 1140.5240 \\
\hline 28 & 10.0000 & 10.0000 & 1140.5240 & 1140.5240 \\
\hline 29 & 10.0000 & 10.0000 & 1140.5240 & 1140.5240 \\
\hline 30 & 87.8008 & 87.8009 & 706.5149 & 706.5162 \\
\hline 31 & 189.9989 & 190.0000 & 1643.9869 & 1643.9913 \\
\hline 32 & 189.9989 & 190.0000 & 1643.9869 & 1643.9913 \\
\hline 33 & 189.9989 & 190.0000 & 1643.9869 & 1643.9913 \\
\hline 34 & 164.8036 & 164.8028 & 1585.6100 & 1585.5961 \\
\hline 35 & 164.8036 & 164.8032 & 1539.9348 & 1539.9274 \\
\hline 36 & 164.8036 & 164.8032 & 1539.9348 & 1539.9274 \\
\hline 37 & 110.0000 & 110.0000 & 1220.1661 & 1220.1661 \\
\hline 38 & 110.0000 & 110.0000 & 1220.1661 & 1220.1661 \\
\hline 39 & 110.0000 & 110.0000 & 1220.1661 & 1220.1661 \\
\hline 40 & 511.2794 & 511.2789 & 5540.9299 & 5540.9289 \\
\hline Sum & 10500.0000 & 10500.0000 & 121415.0522 & 121414.9137 \\
\hline
\end{tabular}

5.4. Test Case 3: Memetic Implementation with Honey Bee Mating Optimization. HBMO algorithm is selected as a final experiment for the memetic implementation in this study. Up until now, the best minimum generation cost of ELD for the same test system using HBMO has been reported as $\$ 121,412.5704$ [34], and each generator's output generation at this point is presented in Table 5. Using a memetic combination to $\mathrm{HBMO}$ (M_HBMO) proposed in this paper,
TABLE 5: Results for Test Case 3.

\begin{tabular}{|c|c|c|c|c|}
\hline \multirow{2}{*}{ Number } & \multicolumn{2}{|c|}{ Generation (MW) } & \multicolumn{2}{|c|}{ Cost $(\$)$} \\
\hline & HBMO [34] & M_HBMO & HBMO [34] & M_HBMO \\
\hline 1 & 110.8018 & 110.8014 & 925.1296 & 925.1218 \\
\hline 2 & 110.8000 & 110.8000 & 925.0998 & 925.0998 \\
\hline 3 & 97.3999 & 97.3999 & 1190.5485 & 1190.5489 \\
\hline 4 & 179.7331 & 179.7331 & 2143.5503 & 2143.5507 \\
\hline 5 & 87.7998 & 87.7999 & 706.5003 & 706.5002 \\
\hline 6 & 140.0000 & 140.0000 & 1596.4643 & 1596.4643 \\
\hline 7 & 259.5997 & 259.5997 & 2612.8846 & 2612.8850 \\
\hline 8 & 284.5997 & 284.5997 & 2779.8368 & 2779.8372 \\
\hline 9 & 284.5997 & 284.5997 & 2798.2303 & 2798.2307 \\
\hline 10 & 130.0000 & 130.0000 & 2502.0650 & 2502.0650 \\
\hline 11 & 94.0000 & 94.0000 & 1893.3054 & 1893.3054 \\
\hline 12 & 94.0000 & 94.0000 & 1908.1668 & 1908.1668 \\
\hline 13 & 214.7598 & 214.7598 & 3792.0700 & 3792.0702 \\
\hline 14 & 394.2794 & 394.2794 & 6414.8604 & 6414.8606 \\
\hline 15 & 394.2794 & 394.2794 & 6436.5863 & 6436.5865 \\
\hline 16 & 394.2794 & 394.2794 & 6436.5863 & 6436.5865 \\
\hline 17 & 489.2794 & 489.2794 & 5296.7107 & 5296.7112 \\
\hline 18 & 489.2794 & 489.2794 & 5288.7652 & 5288.7656 \\
\hline 19 & 511.2794 & 511.2794 & 5540.9292 & 5540.9296 \\
\hline 20 & 511.2794 & 511.2794 & 5540.9092 & 5540.9096 \\
\hline 21 & 523.2794 & 523.2794 & 5071.2897 & 5071.2902 \\
\hline 22 & 523.2807 & 523.2806 & 5071.3156 & 5071.3153 \\
\hline 23 & 523.2794 & 523.2794 & 5057.2231 & 5057.2236 \\
\hline 24 & 523.2794 & 523.2794 & 5057.2231 & 5057.2236 \\
\hline 25 & 523.2794 & 523.2794 & 5275.0885 & 5275.0890 \\
\hline 26 & 523.2794 & 523.2794 & 5275.0885 & 5275.0890 \\
\hline 27 & 10.0000 & 10.0000 & 1140.5240 & 1140.5240 \\
\hline 28 & 10.0000 & 10.0000 & 1140.5240 & 1140.5240 \\
\hline 29 & 10.0000 & 10.0000 & 1140.5240 & 1140.5240 \\
\hline 30 & 87.7999 & 87.7999 & 706.5001 & 706.5005 \\
\hline 31 & 190.0000 & 190.0000 & 1643.9913 & 1643.9913 \\
\hline 32 & 190.0000 & 190.0000 & 1643.9913 & 1643.9913 \\
\hline 33 & 190.0000 & 190.0000 & 1643.9913 & 1643.9913 \\
\hline 34 & 164.8015 & 164.8015 & 1585.5733 & 1585.5732 \\
\hline 35 & 194.3928 & 194.3928 & 1985.3690 & 1985.3693 \\
\hline 36 & 200.0000 & 200.0000 & 2043.7270 & 2043.7270 \\
\hline 37 & 110.0000 & 110.0000 & 1220.1661 & 1220.1661 \\
\hline 38 & 110.0000 & 110.0000 & 1220.1661 & 1220.1661 \\
\hline 39 & 110.0000 & 110.0000 & 1220.1661 & 1220.1661 \\
\hline 40 & 511.2794 & 511.2794 & 5540.9292 & 5540.9296 \\
\hline Sum & 10500.0000 & 10500.0000 & 121412.5704 & 121412.5702 \\
\hline
\end{tabular}

the better minimum cost of the same ELD problem can be obtained as $\$ 121,414.5702$, and the results are presented in Table 5 .

\section{Conclusions}

In summary, this study introduced a memetic scheme of metaheuristic algorithm and gradient-based technique 
TABLE 6: Test results and comparison.

\begin{tabular}{lc}
\hline Algorithm & $\begin{array}{c}\text { Minimum } \\
\text { cost (\$) }\end{array}$ \\
\hline Chaotic differential evolution and quadratic & $121,741.9700$ \\
programming [54] & $121,735.4700$ \\
PSO [55] & $121,698.5100$ \\
Hybrid differential evolution [56] & $121,664.4300$ \\
New PSO [57] & $121,663.5200$ \\
Antipredatory PSO [55] & $121,501.1400$ \\
Self-organizing hierarchical PSO [58] & $121,479.5000$ \\
Biography-based optimization [59] & $121,458,0000$ \\
GA-PS-SQP [60] & $121,423.6300$ \\
Bacterial foraging [61] & $121,415.4560$ \\
Harmony search (HS) [36] & $\mathbf{1 2 1 , 4 1 5 . 4 5 2 5}$ \\
Memetic harmony search (this study) & $121,415.1400$ \\
Pattern search [62] & $121,415.0522$ \\
Firefly algorithm (FA) [33] & $\mathbf{1 2 1 , 4 1 4 . 9 1 3 7}$ \\
Memetic firefly algorithm (this study) & $121,412.5704$ \\
Honey bee mating optimization (HBMO) [34] & $\mathbf{1 2 1 , 4 1 2 . 5 7 0 2}$ \\
Memetic HBMO (this study) &
\end{tabular}

and then applied it to a popular benchmark problem of 40 generating-unit ELD problem with valve-point loading, obtaining better solutions than other solutions ever found in the literature. As shown in Table 6, the result ( $\$ 121415.4560)$ of HS was further improved into $\$ 121415.4525$ as shown in bold font; that (\$121415.0522) of FA was further improved into $\$ 121414.9137$ as shown in bold font; and that (\$121412.5704) of HBMO was further improved into \$121412.5702 as shown in bold font. We have extended the simulation comparisons against the other recent metaheuristic approaches, and it is also listed in Table 6. We also hope that this table and corresponding references become a good literature survey for future researches.

While metaheuristic algorithms perform well in global search, they do not perform well in local search. On the contrary, while gradient-based techniques perform well in local search, they do not perform well in global search. Thus, the memetic approach in this study can be mutually complementary to obtain better solutions than either metaheuristiconly solution or gradient-based-only solution. Not only this ELD problem, but also hydrologic flood model calibration had better results using the memetic approach [79].

With this successful approach, we would like to explore more complex real-world ELD problems as well as other optimization problems. The proposed method can be applied to improve the simulation results of existing approaches $[80$, 81] in the future.

\section{Conflict of Interests}

The authors declare that there is no conflict of interests regarding the publication of this paper.

\section{Acknowledgment}

This work was supported by the Gachon University Research Fund of 2013 (GCU-2013-R194).

\section{References}

[1] J. F. Bard, "Short-term scheduling of thermal-electric generators using Lagrangian relaxation,” Operations Research, vol. 36, no. 5, pp. 756-766, 1988.

[2] Z. X. Liang and J. D. Glover, "A zoom feature for a dynamic programming solution to economic dispatch including transmission losses," IEEE Transactions on Power Systems, vol. 7, no. 2, pp. 544-550, 1992.

[3] C. L. Chen and S. C. Wang, "Branch-and-bound scheduling for thermal generating units," IEEE Transactions on Energy Conversion, vol. 8, no. 2, pp. 184-189, 1993.

[4] J. Nanda, L. Hari, and M. L. Kothari, "Economic emission load dispatch with line flow constraints using a classical technique," IET Generation, Transmission and Distribution, vol. 141, no. 1, pp. 1-10, 1994.

[5] P. G. Lowery, "Generating unit commitment by dynamic programming," IEEE Transactions on Power Apparatus and Systems, vol. 85, no. 5, pp. 422-426, 1996.

[6] J. P. D. Chattopadhyay, "A multi-area linear programming approach for analysis of economic operation of the Indian power system," IEEE Transactions on Power Systems, vol. 11, no. 1, pp. 52-58, 1996.

[7] J. Y. Fan and L. Zhang, "Real-time economic dispatch with line flow and emission constraints using quadratic programming," IEEE Transactions on Power Systems, vol. 13, no. 2, pp. 320-325, 1998.

[8] D. C. Walters and G. B. Sheble, "Genetic algorithm solution of economic dispatch with value point loading," IEEE Transactions on Power Systems, vol. 8, no. 3, pp. 1325-1332, 1993.

[9] P. H. Chen and H. C. Chang, "Large-scale economic dispatch by genetic algorithm," IEEE Transactions on Power Systems, vol. 10, no. 4, pp. 1919-1926, 1995.

[10] G. B. Sheble and K. Brittig, "Refined genetic algorithmeconomic dispatch example," IEEE Transactions on Power Systems, vol. 10, no. 1, pp. 117-124, 1995.

[11] K. S. Swarup and S. Yamashiro, "Unit commitment solution methodology using genetic algorithm," IEEE Transactions on Power Systems, vol. 17, no. 1, pp. 87-91, 2002.

[12] I. G. Damousis, A. G. Bakirtzis, and P. S. Dokopoulos, "Network-constrained economic dispatch using real-coded genetic algorithm," IEEE Transactions on Power Systems, vol. 18, no. 1, pp. 198-205, 2003.

[13] C. L. Chiang, "Improved genetic algorithm for power economic dispatch of units with valve-point effects and multiple fuels," IEEE Transactions on Power Systems, vol. 20, no. 4, pp. 16901699, 2005.

[14] C. L. Chiang, "Genetic-based algorithm for power economic load dispatch," IET Generation, Transmission and Distribution, vol. 1, no. 2, pp. 261-269, 2007.

[15] S. Kumar and R. Naresh, "Nonconvex economic load dispatch using an efficient real-coded genetic algorithm," Applied Soft Computing Journal, vol. 9, no. 1, pp. 321-329, 2009.

[16] H. T. Yang, P. C. Yang, and C. L. Huang, "Evolutionary programming based economic dispatch for units with non-smooth fuel cost functions," IEEE Transactions on Power Systems, vol. 11, no. 1, pp. 112-118, 1996. 
[17] K. P. Wong and J. Yuryevich, "Evolutionary-programmingbased algorithm for environmentally-constrained economic dispatch," IEEE Transactions on Power Systems, vol. 13, no. 2, pp. 301-306, 1998.

[18] N. Sinha, R. Chakrabarti, and P. K. Chattopadhyay, "Evolutionary programming techniques for economic load dispatch," IEEE Transactions on Evolutionary Computation, vol. 7, no. 1, pp. 8394, 2003.

[19] A. Pereira-Neto, C. Unsihuay, and O. R. Saavedra, "Efficient evolutionary strategy optimization procedure to solve the nonconvex economic dispatch problem with generator constraints," IET Generation, Transmission and Distribution, vol. 152, no. 5, pp. 653-660, 2005.

[20] Z. L. Gaing, "Particle swarm optimization to solving the economic dispatch considering the generator constraints," IEEE Transactions on Power Systems, vol. 18, no. 3, pp. 1187-1195, 2003.

[21] J. B. Park, K. S. Lee, J. R. Shin, and K. Y. Lee, "A particle swarm optimization for economic dispatch with nonsmooth cost functions," IEEE Transactions on Power Systems, vol. 20, no. 1, pp. 34-42, 2005.

[22] J. Cai, X. Ma, L. Li, and P. Haipeng, "Chaotic particle swarm optimization for economic dispatch considering the generator constraints," Energy Conversion and Management, vol. 48, no. 2, pp. 645-653, 2007.

[23] A. I. Selvakumar and K. Thanushkodi, "A new particle swarm optimization solution to nonconvex economic dispatch problems," IEEE Transactions on Power Systems, vol. 22, no. 1, pp. 42-51, 2007.

[24] K. T. Chaturvedi, M. Pandit, and L. Srivastava, "Self-organizing hierarchical particle swarm optimization for nonconvex economic dispatch," IEEE Transactions on Power Systems, vol. 23, no. 3, pp. 1079-1087, 2008.

[25] A. I. Selvakumar and K. Thanushkodi, "Anti-predatory particle swarm optimization: solution to nonconvex economic dispatch problems," Electric Power Systems Research, vol. 78, no. 1, pp. 2$10,2008$.

[26] K. T. Chaturvedi, M. Pandit, and L. Srivastava, "Particle swarm optimization with crazy particles for nonconvex economic dispatch," Applied Soft Computing Journal, vol. 9, no. 3, pp. 962969, 2009.

[27] J. G. Vlachogiannis and K. Y. Lee, "Economic load dispatcha comparative study on heuristic optimization techniques with an improved coordinated aggregation-based PSO," IEEE Transactions on Power Systems, vol. 24, no. 2, pp. 991-1001, 2009.

[28] S. S. S. Hosseini and A. H. Gandomi, "Discussion of "Economic load dispatch-a comparative study on heuristic optimization techniques with an improved coordinated aggregation-based PSO'”' IEEE Transactions on Power Systems, vol. 25, no. 1, p. 590, 2010.

[29] W. M. Lin, F. S. Cheng, and M. T. Tsay, "An improved tabu search for economic dispatch with multiple minima," IEEE Transactions on Power Systems, vol. 17, no. 1, pp. 108-112, 2002.

[30] D. Liu and Y. Cai, "Taguchi method for solving the economic dispatch problem with nonsmooth cost functions," IET Generation, Transmission and Distribution, vol. 1, no. 5, pp. 793-803, 2007.

[31] B. K. Panigrahi and V. R. Pandi, "Bacterial foraging optimisation: Nelder-Mead hybrid algorithm for economic load dispatch," IET Generation, Transmission and Distribution, vol. 2, no. 4, pp. 556-565, 2008.

[32] A. Bhattacharya and P. K. Chattopadhyay, "Solving complex economic load dispatch problems using biogeography-based optimization," Expert Systems with Applications, vol. 37, no. 5, pp. 3605-3615, 2010.

[33] X. S. Yang, S. S. S. Hosseini, and A. H. Gandomi, "Firefly algorithm for solving non-convex economic dispatch problems with valve loading effect," Applied Soft Computing Journal, vol. 12, no. 3, pp. 1180-1186, 2012.

[34] T. Niknam, H. D. Mojarrad, H. Z. Meymand, and B. B. Firouzi, "A new honey bee mating optimization algorithm for nonsmooth economic dispatch," Energy, vol. 36, no. 2, pp. 896-908, 2011.

[35] L. D. S. Coelho and V. C. Mariani, "An improved harmony search algorithm for power economic load dispatch," Energy Conversion and Management, vol. 50, no. 10, pp. 2522-2526, 2009.

[36] B. K. Panigrahi, V. R. Pandi, S. Das, Z. Cui, and R. Sharma, "Economic load dispatch using population-variance harmony search algorithm," Transactions of the Institute of Measurement and Control, vol. 34, no. 6, pp. 746-754, 2011.

[37] K. P. Wong and Y. W. Wong, "Thermal generator scheduling using hybrid genetic/simulated-annealing approach," IET Generation, Transmission and Distribution, vol. 142, no. 4, pp. 372380, 1995.

[38] L. D. S. Coelho and V. C. Mariani, "Combining of chaotic differential evolution and quadratic programming for economic dispatch optimization with valve-point effect," IEEE Transactions on Power Systems, vol. 21, no. 2, pp. 989-996, 2006.

[39] L. D. S. Coelho and V. C. Mariani, "Erratum correction to "Combining of chaotic differential evolution and quadratic programming for economic dispatch optimization with valve point effect"', IEEE Transactions on Power Systems, vol. 21, no. 3, p. 1465, 2006.

[40] S. K. Wang, J. P. Chiou, and C. W. Liu, "Non-smooth/nonconvex economic dispatch by a novel hybrid differential evolution algorithm," IET Generation, Transmission and Distribution, vol. 1, no. 5, pp. 793-803, 2007.

[41] J. S. Alsumait, J. K. Sykulski, and A. K. Al-Othman, "A hybrid GA-PS-SQP method to solve power system valve-point economic dispatch problems," Applied Energy, vol. 87, no. 5, pp. 1773-1781, 2010.

[42] H. Altun and T. Yalcinoz, "Implementing soft computing techniques to solve economic dispatch problem in power systems," Expert Systems with Applications, vol. 35, no. 4, pp. 1668-1678, 2008.

[43] Z. W. Geem and Y. H. Cho, "Handling non-convex heat-power feasible region in combined heat and power economic dispatch," International Journal of Electrical Power and Energy Systems, vol. 34, no. 1, pp. 171-173, 2012.

[44] M. H. Moradi and M. Abedini, "A combination of genetic algorithm and particle swarm optimization for optimal DG location and sizing in distribution systems," International Journal of Electrical Power and Energy Systems, vol. 34, no. 1, pp. 66-74, 2012.

[45] A. Mahor and S. Rangnekar, "Short term generation scheduling of cascaded hydro electric system using novel self adaptive inertia weight PSO," International Journal of Electrical Power and Energy Systems, vol. 34, no. 1, pp. 1-9, 2012.

[46] Z. W. Geem, "Size optimization for a hybrid photovoltaicwind energy system," International Journal of Electrical Power \& Energy Systems, vol. 42, no. 1, pp. 448-451, 2012.

[47] A. Bhattacharya and P. K. Chattopadhyay, "Solution of economic power dispatch problems using oppositional 
biogeography-based optimization," Electric Power Components and Systems, vol. 38, no. 10, pp. 1139-1160, 2010.

[48] J. G. Vlachogiannis and K. Y. Lee, "Economic load dispatcha comparative study on heuristic optimization techniques with an improved coordinated aggregation-based PSO," IEEE Transactions on Power Systems, vol. 24, no. 2, pp. 991-1001, 2009.

[49] K. T. Chaturvedi, M. Pandit, and L. Srivastava, "Self-organizing hierarchical particle swarm optimization for nonconvex economic dispatch," IEEE Transactions on Power Systems, vol. 23, no. 3, pp. 1079-1087, 2008.

[50] B. K. Panigrahi, V. Ravikumar Pandi, and S. Das, "Adaptive particle swarm optimization approach for static and dynamic economic load dispatch," Energy Conversion and Management, vol. 49, no. 6, pp. 1407-1415, 2008.

[51] C. C. Kuo, "A novel coding scheme for practical economic dispatch by modified particle swarm approach," IEEE Transactions on Power Systems, vol. 23, no. 4, pp. 1825-1835, 2008.

[52] A. I. Selvakumar and K. Thanushkodi, "A new particle swarm optimization solution to nonconvex economic dispatch problems," IEEE Transactions on Power Systems, vol. 22, no. 1, pp. 42-51, 2007.

[53] B. K. Panigrahi and V. Ravikumar Pandi, "Bacterial foraging optimisation: Nelder-Mead hybrid algorithm for economic load dispatch," IET Generation, Transmission and Distribution, vol. 2, no. 4, pp. 556-565, 2008.

[54] L. D. S. Coelho and V. C. Mariani, "Combining of chaotic differential evolution and quadratic programming for economic dispatch optimization with valve-point effect," IEEE Transactions on Power Systems, vol. 21, no. 2, pp. 989-996, 2006.

[55] A. I. Selvakumar and K. Thanushkodi, "Anti-predatory particle swarm optimization: solution to nonconvex economic dispatch problems," Electric Power Systems Research, vol. 78, no. 1, pp. 2$10,2008$.

[56] S. K. Wang, J. P. Chiou, and C. W. Liu, "Non-smooth/nonconvex economic dispatch by a novel hybrid differential evolution algorithm," IET Generation, Transmission and Distribution, vol. 1, no. 5, pp. 793-803, 2007.

[57] A. I. Selvakumar and K. Thanushkodi, "A new particle swarm optimization solution to nonconvex economic dispatch problems," IEEE Transactions on Power Systems, vol. 22, no. 1, pp. 42-51, 2007.

[58] K. T. Chaturvedi, M. Pandit, and L. Srivastava, "Self-organizing hierarchical particle swarm optimization for nonconvex economic dispatch," IEEE Transactions on Power Systems, vol. 23, no. 3, pp. 1079-1087, 2008.

[59] A. Bhattacharya and P. K. Chattopadhyay, "Solving complex economic load dispatch problems using biogeography-based optimization," Expert Systems with Applications, vol. 37, no. 5, pp. 3605-3615, 2010.

[60] J. S. Alsumait, J. K. Sykulski, and A. K. Al-Othman, "A hybrid GA-PS-SQP method to solve power system valve-point economic dispatch problems," Applied Energy, vol. 87, no. 5, pp. 1773-1781, 2010.

[61] B. K. Panigrahi and V. R. Pandi, "Bacterial foraging optimisation: Nelder-Mead hybrid algorithm for economic load dispatch," IET Generation, Transmission and Distribution, vol. 2, no. 4, pp. 556-565, 2008.

[62] J. S. Al-Sumait, A. K. AL-Othman, and J. K. Sykulski, "Application of pattern search method to power system valve-point economic load dispatch," International Journal of Electrical Power and Energy Systems, vol. 29, no. 10, pp. 720-730, 2007.
[63] F. Neri and C. Cotta, "Memetic algorithms and memetic computing optimization: a literature review," Swarm and Evolutionary Computation, vol. 2, pp. 1-14, 2012.

[64] J. S. Al-Sumait, A. K. AL-Othman, and J. K. Sykulski, "Application of pattern search method to power system valve-point economic load dispatch," International Journal of Electrical Power and Energy Systems, vol. 29, no. 10, pp. 720-730, 2007.

[65] G. Iacca, F. Neri, E. Mininno, Y. S. Ong, and M. H. Lim, "Ockham's Razor in memetic computing: three stage optimal memetic exploration," Information Sciences, vol. 188, pp. 17-43, 2012.

[66] F. Caraffini, F. Neri, G. Iacca, and A. Mol, "Parallel memetic structures," Information Sciences, vol. 227, pp. 60-82, 2013.

[67] H. Ishibuchi, T. Yoshida, and T. Murata, "Balance between genetic search and local search in memetic algorithms for multiobjective permutation flowshop scheduling," IEEE Transactions on Evolutionary Computation, vol. 7, no. 2, pp. 204-223, 2003.

[68] K. C. Tan, S. C. Chiam, A. A. Mamun, and C. K. Goh, "Balancing exploration and exploitation with adaptive variation for evolutionary multi-objective optimization," European Journal of Operational Research, vol. 197, no. 2, pp. 701-713, 2009.

[69] H. A. Abbass, "An evolutionary artificial neural networks approach for breast cancer diagnosis," Artificial Intelligence in Medicine, vol. 25, no. 3, pp. 265-281, 2002.

[70] J. Tang, M. H. Lim, and Y. S. Ong, "Diversity-adaptive parallel memetic algorithm for solving large scale combinatorial optimization problems," Soft Computing, vol. 11, no. 9, pp. 873-888, 2007.

[71] S. M. K. Hasan, R. Sarker, D. Essam, and D. Cornforth, "Memetic algorithms for solving job-shop scheduling problems," Memetic Computing, vol. 1, no. 1, pp. 69-83, 2009.

[72] K. K. Lim, Y. S. Ong, M. H. Lim, X. Chen, and A. Agarwal, "Hybrid ant colony algorithms for path planning in sparse graphs," Soft Computing, vol. 12, no. 10, pp. 981-994, 2008.

[73] K. C. Tan, C. Y. Cheong, and C. K. Goh, "Solving multiobjective vehicle routing problem with stochastic demand via evolutionary computation," European Journal of Operational Research, vol. 177, no. 2, pp. 813-839, 2006.

[74] A. Caponio, G. L. Cascella, F. Neri, N. Salvatore, and M. Sumner, "A fast adaptive memetic algorithm for online and offline control design of PMSM drives," IEEE Transactions on Systems, Man, and Cybernetics B, vol. 37, no. 1, pp. 28-41, 2007.

[75] F. Neri and E. Mininno, "Memetic compact differential evolution for cartesian robot control," IEEE Computational Intelligence Magazine, vol. 5, no. 2, pp. 54-65, 2010.

[76] T. Rogalsky and R. W. Derksen, "Hybridization of differential evolution for aerodynamic design," in Proceedings of the 8th Annual Conference of the Computational Fluid Dynamics Society of Canada, pp. 729-736, 2000.

[77] Z. W. Geem, "Parameter estimation for the nonlinear Muskingum model using the BFGS technique," Journal of Irrigation and Drainage Engineering, vol. 132, no. 5, pp. 474-478, 2006.

[78] Z. W. Geem, "Parameter estimation of the nonlinear muskingum model using parameter-setting-free harmony search," Journal of Hydrologic Engineering, vol. 16, no. 8, pp. 684-688, 2011.

[79] H. Karahan, G. Gurarslan, and Z. W. Geem, "Parameter estimation of the nonlinear Muskingum flood routing model using a hybrid harmony search algorithm," ASCE Journal of Hydrologic Engineering, vol. 18, no. 3, pp. 352-260, 2013. 
[80] F. Gao and L. Han, "Implementing the Nelder-Mead simplex algorithm with adaptive parameters," Computational Optimization and Applications, vol. 51, no. 1, pp. 259-277, 2012.

[81] E. Afzalan and M. Joorabian, "Emission, reserve and economic load dispatch problem with non-smooth and non-convex cost functions using epsilon-multi-objective genetic algorithm variable," International Journal of Electrical Power and Energy Systems, vol. 52, pp. 55-67, 2013. 


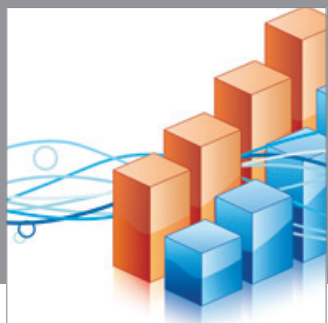

Advances in

Operations Research

mansans

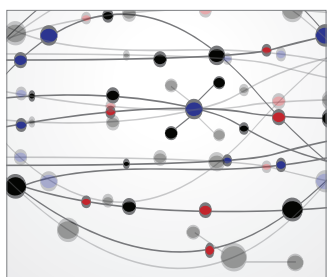

The Scientific World Journal
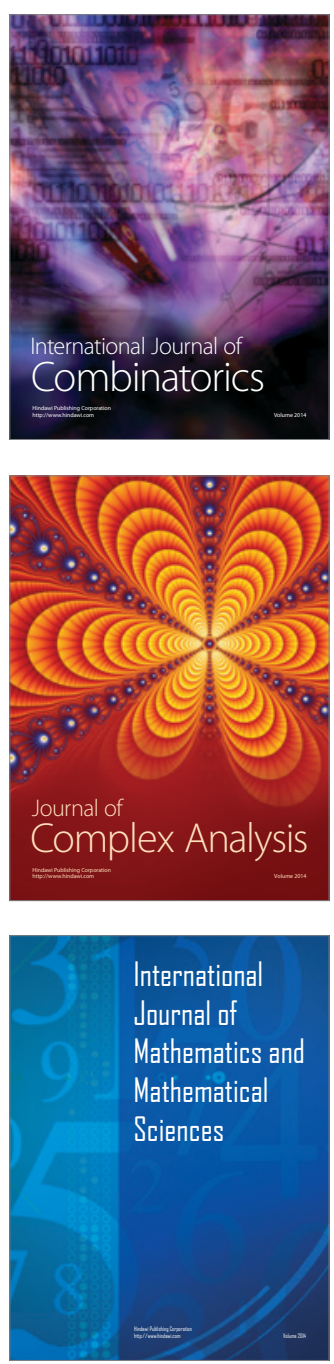
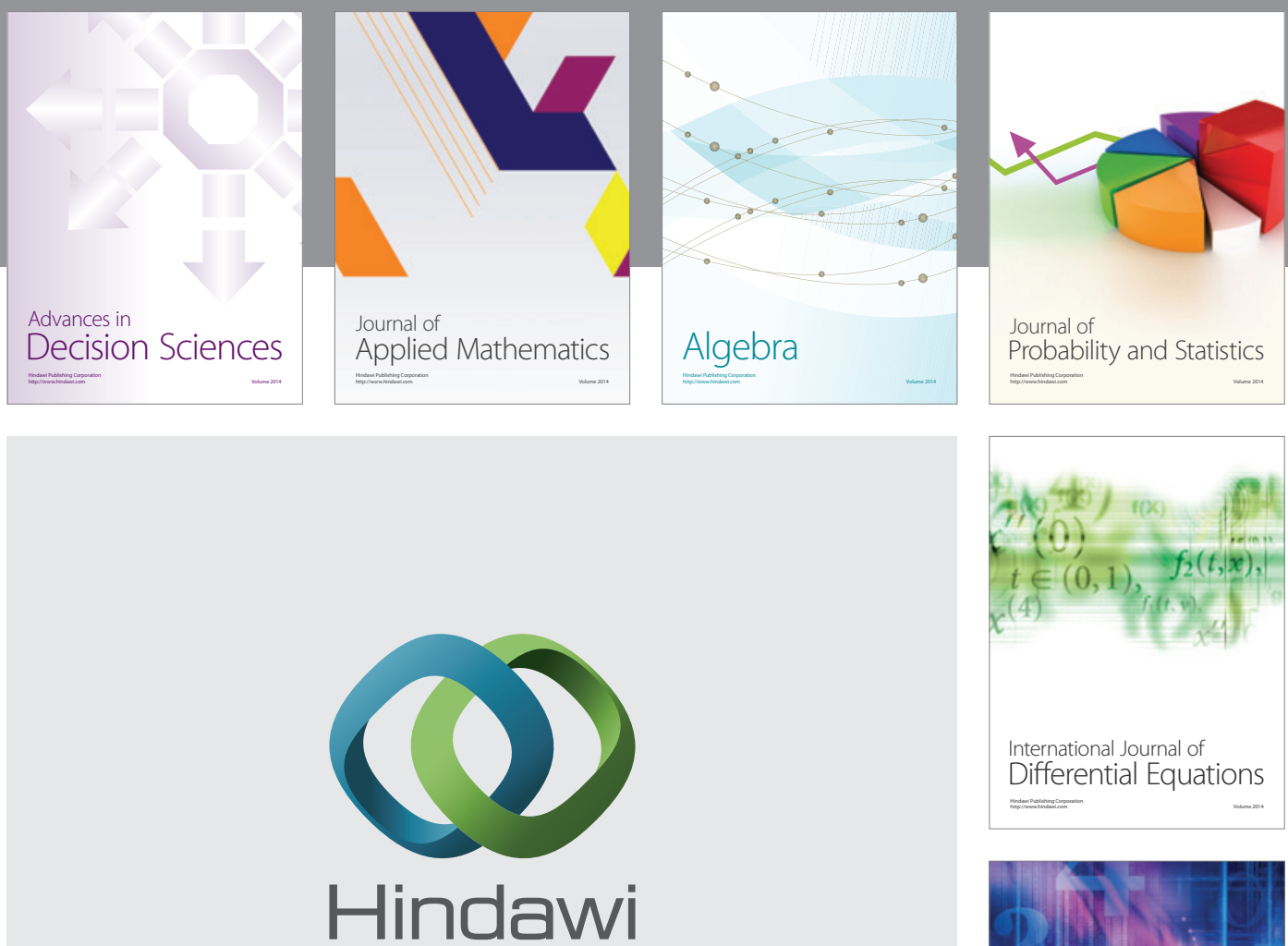

Submit your manuscripts at http://www.hindawi.com
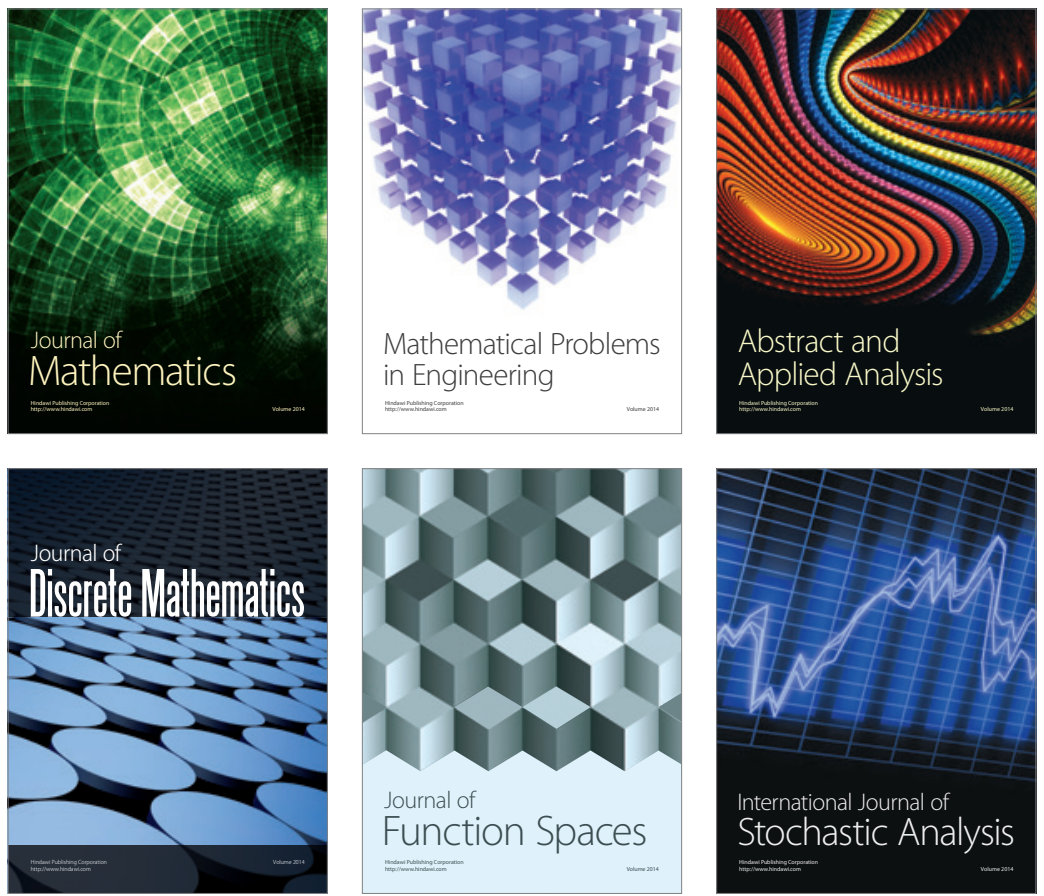

Journal of

Function Spaces

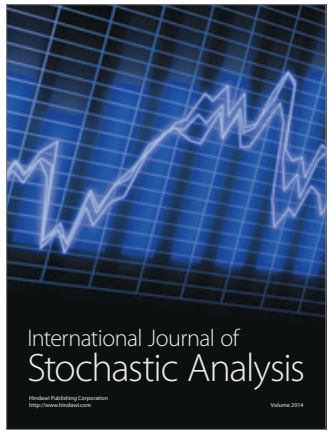

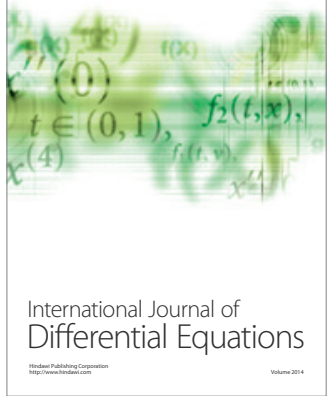
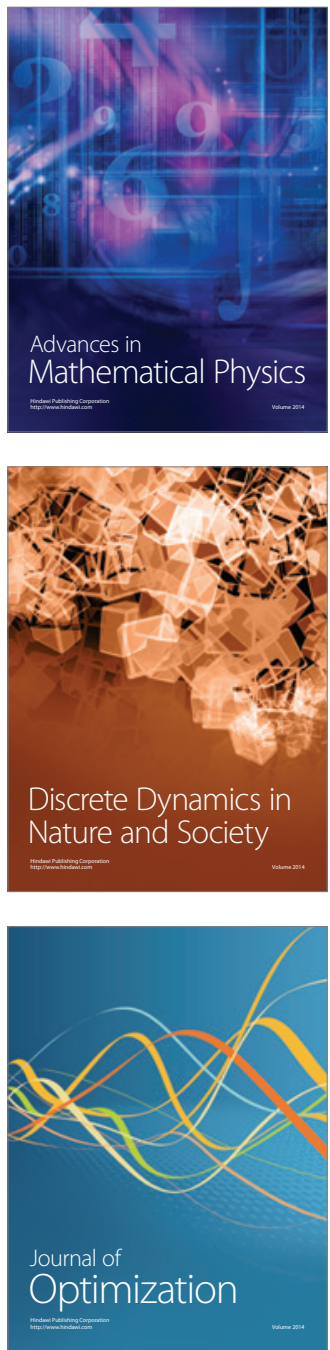